\title{
Development of the opto-fluidic servo valve
}

\author{
Dai Kanai*,Satoru Nakayama*,Osamu Oyama*, \\ Toshihiko Yoshimitsu**and Keijiro Yamamoto** \\ *Meiji University, 1-1-1 Higasimita, Tama-ku, Kawasaki-shi 214, Japan \\ **Kanagawa Institute of Technology,1033 Shimoogino, Atugi-shi, 243-02, Japan
}

\begin{abstract}
An opto-pneumatic servo system employing opto-fluidic converter and pneumatic valve can operate the mechanical system directed by optical signal and work in hazardous environments because it does not use the electric immediate signal. The converter transforms the optical signal intensity into proportional fluid power, i.e. pneumatic pressure, although the pressure is still weak and is not enough to operate the mechanical system directly. In this report, as the practical system, the opto-pneumatic servo valve is developed.

The output pressure of Opto-fluidic converter is enlarged proportionally in the serial stages of fluidic amplifier and led to pneumatical input of servo valve as a pilot signal which excite the both sides of spool in the valve. The study is mainly carried out for optimizing the design of serial stage of fluidic amplifier, in order to generates the enough high output pressure which can be used as the pilot (operate) pressure in the pneumatic proportional valve. The performances and applications of the system will be shown.
\end{abstract}

\section{KEYWORDS}

Optical servo valve, Laminar proportional amplifier (LPA), Opto-pneumatic control system

\section{Introduction}

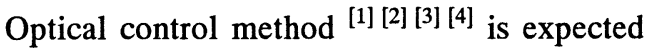
as a system working in the field affected by electromagnetic noises, and having high-speed responsibility, and is excellent in safety. Furthermore it can be compact structure and is light weight.

In order to employ the feature of the opto-fluidic control system, the source of power must depend on fluid alone and the system must not have an electronic circuit in moving device.

The system is realized by using opt-fluidic converter which transforms an optical signal into a pneumatic pressure signal without an intermediate electric signal. And so we developed an opt-fluidic servo system combining the opto-fluidic converter and a pneumatic valve in order to directly operate the pneumatic actuator by using without any electric devices.

\section{Purpose of the research}

In this research, we developed the analog servo valve "an optical servo valve" which has the optical input and the enough high output pressure, combining with the opto-fluidic converter and an air pilot servo valve. The characteristic of this optical servo valve is investigated, and pressure control is performed.

Furthermore we verify about the usefulness of an optical servo valve by the result. 


\section{The opto-fluidic servo valve configuration}

\subsection{Systems configuration}

Fig.1 shows systems configuration. The analog signal sent from computer is inputted into a laser diode controller, and the optical power of a laser diode is determined. An optical input is transformed into the pressure signal with opto-fluidic converter, and the pressure is amplified by the LPA (Laminar Proportionality Amplification) cascaded amplifiers, and then amplified pressure signal is inputted into a pneumatic servo valve as a pilot.

A spool inside a servo valve displaces in proportion to this pressure signal, and the position determines the output flow rate or pressure of a servo valve output. The portion surrounded by the dotted line in Figure 1 is an opto-fluidic servo valve part.

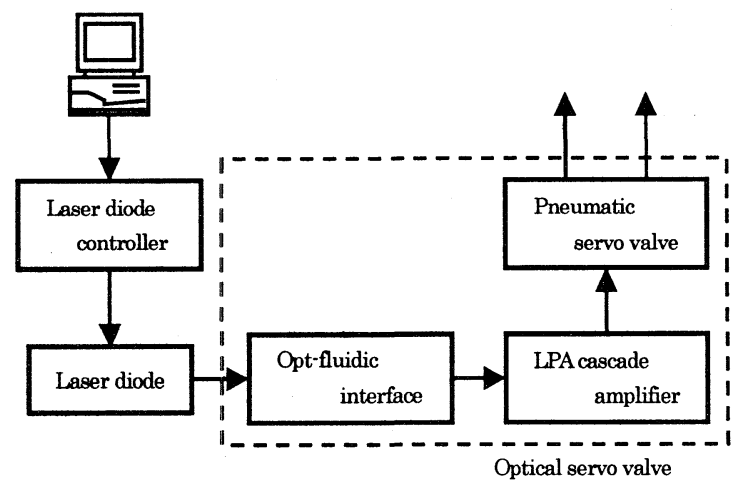

Figure1 Optical servo valve system

\subsection{Opt-fluidic converter}

As the method of changing an optical signal into a fluid signal without a mechanical drive, this research suggests the following system.

(1) An optical absorber places the supply nozzle side of ordinary LPA.

(2) Optical energy is transformed into thermal energy by irradiating an optical signal at the optical absorber through an optical fiber.
(3) A jet stream is deflected when thermal energy affects the temperature distribution of the main jet stream boundary layer.

Since the output of this conversion element is feeble, the more two stages LPA are used to amplify the output in fluid, where the aspect ratio of a supply nozzle is 1.3 of the 1st stage and the 2 nd stage, and is 0.66 of the 3rd step .

An outline figure is shown in Figure2.

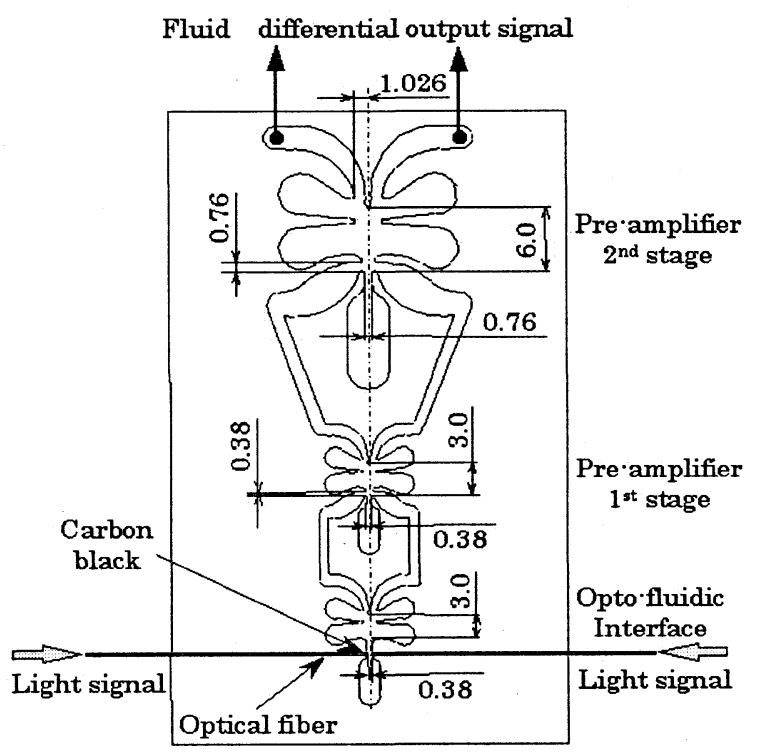

Figure2 Opto-fluidic converter

\subsection{3-stage of LPA cascade amplifier}

Since the output of optical-fluid converter does not have sufficient power for making the spool of the servo valve drive, the output pressure of converter is boosted with 3-stage of LPA cascade. Although each LPA of cascade amplifier is the same shape and same aspect ratio (1.03), each stage of the cascade is connected with different number of parallel LPA, i.e. two for 1 st stage, three for 2 nd, and five for 3rd, respectively. The figure is shown in Figure3. 


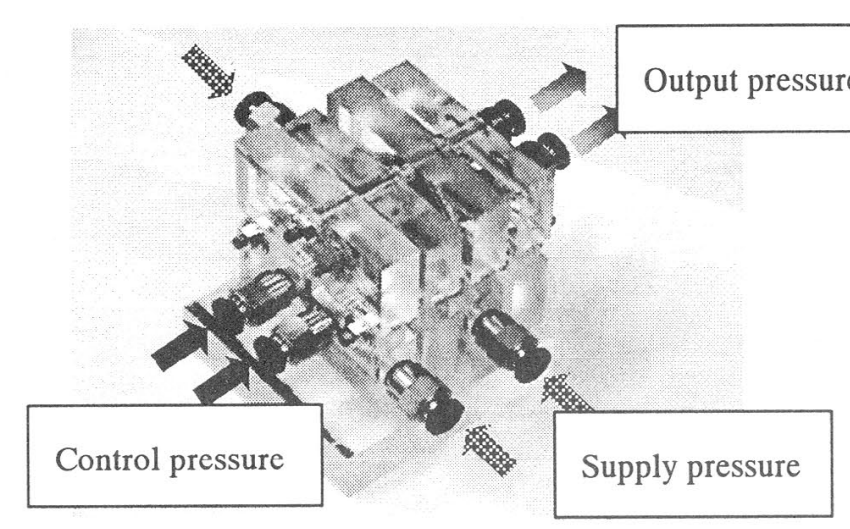

Figure 3 3-stage of LPA cascade amplifier

\subsection{Air servo valve}

Although ordinary servo valve is driven by the input of an electric signal, the pilot type is used in this report. The pressure difference of an input room on either side serves as an input force motor, and when the spool of servo valve moves to right or left, the effective area of output is adjusted. Air servo valve drives when differential pressure is $700[\mathrm{~Pa}]$

A figure of outline is shown in Figure4.

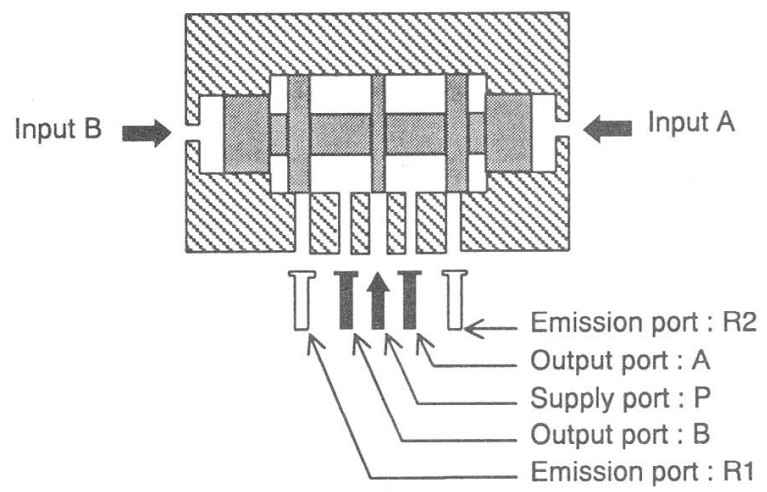

Figure4 Schema of servo valve piloted by air

pressure

\section{The characteristic of a component}

\subsection{The characteristic of opt-fluidic converter and a 3-stage cascade}

The conversion characteristic of optical-fluidic converter is shown in Figure5 (a)(b).

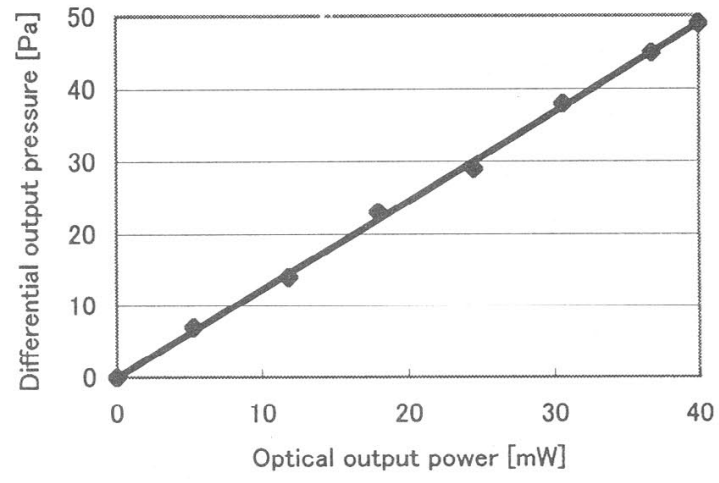

(a) Output A

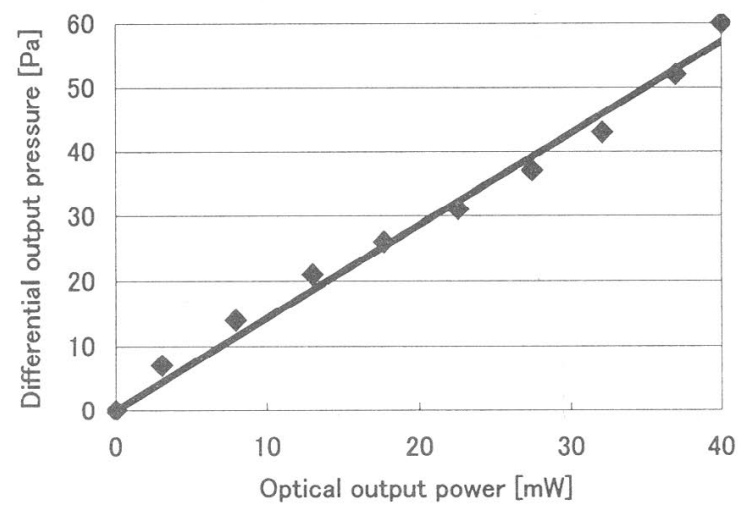

(b) Output B

Figure5 Characteristics of Opto-fluidic interface

At the supply pressure $\mathrm{P}=200,200,500[\mathrm{~Pa}]$, the A port outputs the maximum gain of 1.23 $[\mathrm{Pa} / \mathrm{mW}]$, and the $\mathrm{B}$ port outputs $1.43[\mathrm{~Pa} / \mathrm{mW}]$. Where, a gain is the ratio of the consumption power of the laser diode between the output pressure difference. Moreover, the intensity of an optical input signal and the output pressure of a 3-stage cascade are proportional relations, and at the supply pressure $\mathrm{P}=1.8,6.0$, and $15.0[\mathrm{kPa}]$, the maximum gain of the $\mathrm{A}$ port $21.95[\mathrm{~Pa} / \mathrm{mW}]$ and the $B$ port $24.23[\mathrm{~Pa} / \mathrm{mW}]$ is obtained, and both the maximum differential pressure becomes $950[\mathrm{~Pa}]$. 
The flow rate outputted from the 3-stage of LPA cascade is used to carrying out drive control of the spool of the air servo valve, therefore, in order to increase flow rate, the number of connected LPA in parallel in each step, is increased from $2,2,3$ pieces to $2,3,5$ pieces.

The comparison of the differential flow rate outputted from a 3-stage of LPA cascade is shown in Figure6. The above result shows that the differential input pressure to a 3-stage cascade and the flow rate outputted to A and B port have proportional relation characteristics.

Moreover, it is shown that the output flow rate is increased by lamination of LPA. From the relation between output pressure and flow rate, the maximum output power of optical-fluid conversion system is $71.85[\mathrm{~mW}]$.

\subsection{The characteristic of a 4-stage of LPA cascade}

Because it is possible to have made the spool of an air servo valve drive also with the differential output pressure from a 3-stage of LPA cascade, in order to improve the response of a spool further, the LPA amplification step is increased to 4-stage.

The static characteristic of this 4-stage of LPA cascade is shown in Figure7 (a)(b). Where, the supply pressure of $\mathrm{P}=1 \cdot 5,3 \cdot 0,6 \cdot 0,15.0[\mathrm{kPa}]$ is given from the 1 st to the 4 th stage of a cascade. The differential output pressure becomes a maximum of $950[\mathrm{~Pa}]$ in the 3-stage cascade amplified to a maximum of 1750 [Pa] in 4-stage. While, the differential output pressure gain from the 4-stage cascade by laser $\mathrm{A}$ irradiation is $43.08[\mathrm{~Pa} / \mathrm{mW}]$, and the pressure by laser $B$ is $45.64[\mathrm{~Pa} / \mathrm{mW}]$.

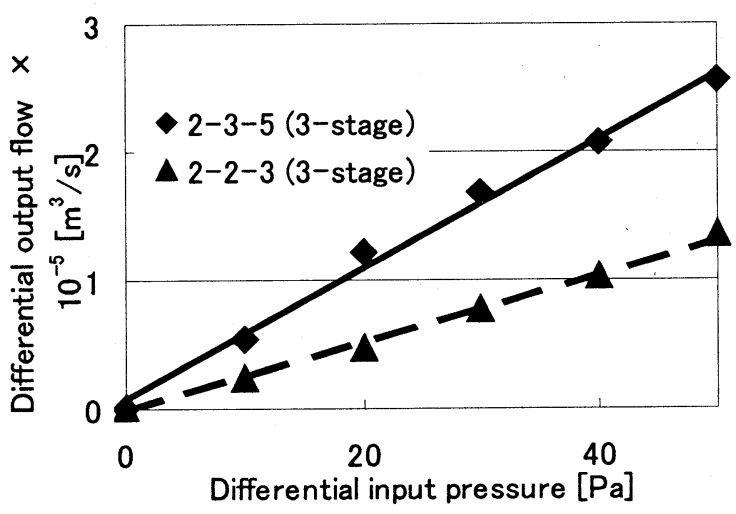

Figure6 Comparison of differential output flow

rate

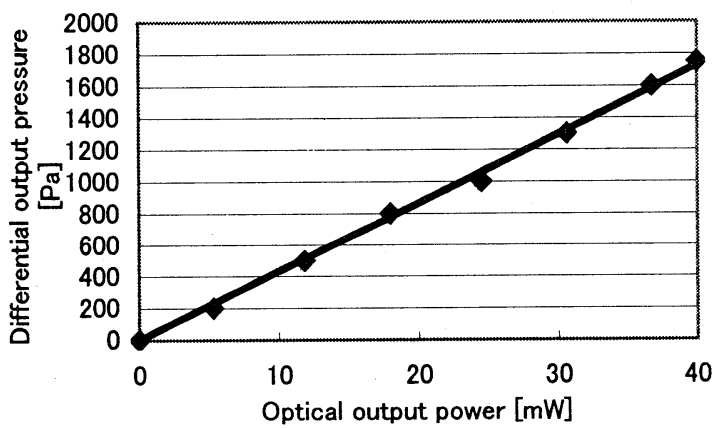

(a)Output A

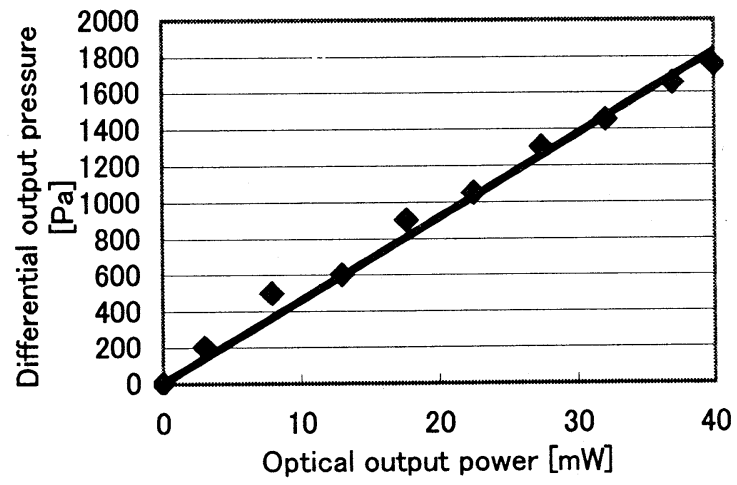

(b)Output B

Figure.7 Characteristics of LPA 4-stage amplifier 


\subsection{The characteristic of an air servo valve}

When inputting a fixed pressure difference into the input parts $A$ and $B$ in the shape of a step wave, the transient response of output pressure value of A port (a) and B (b) port is shown in Figure8. (Supply pressure of an air servo valve is $200[\mathrm{kPa}])$. Here, the dynamic characteristics are compared, where the maximum output of 3-stage cascade is $950[\mathrm{~Pa}]$ and the maximum output of 4-stage cascade is $1750[\mathrm{~Pa}]$. From the figure, the time until the spool of 3-stage cascade and the 4-satage cascade begins to move is compared, and the

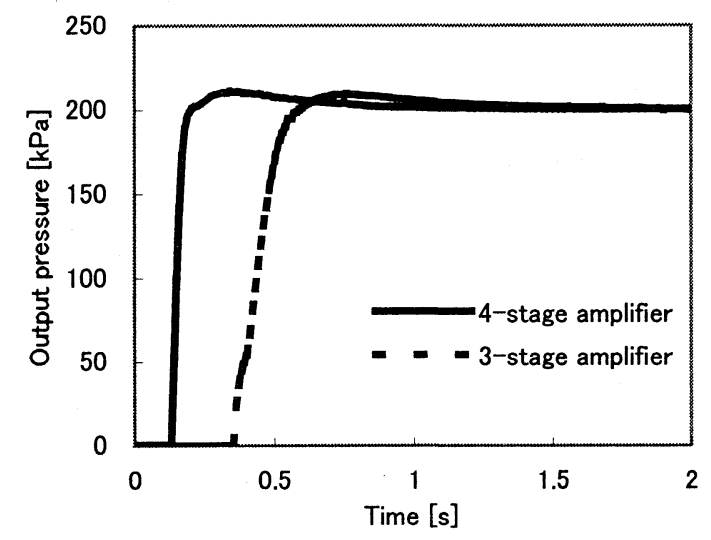

(a) Output port $\mathrm{A}$

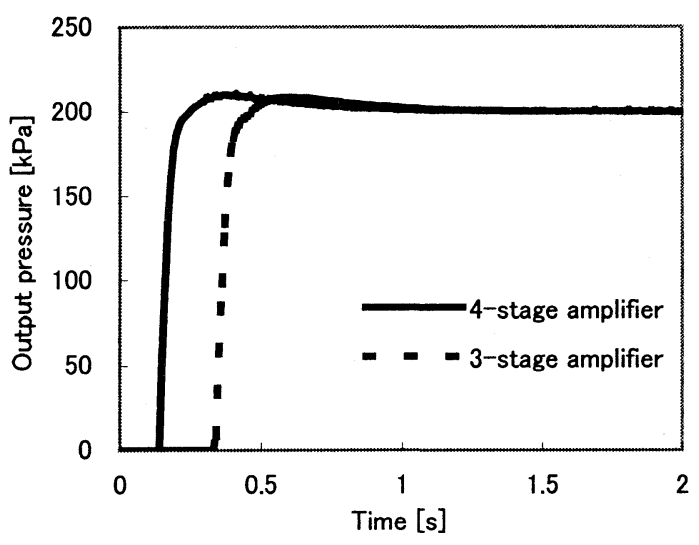

(b) Output port B

Figure8 Step response of optical servo valve response of A port side can be reduced about $57 \%$ of time and B port side can be about $40 \%$.

\section{Pressure control of the optical servo valve}

As an application, we performed output pressure control of air servo valve. The position of the spool in the air servo valve is controlled by PID control.

\subsection{The PID control method}

At the time $\boldsymbol{k}$, a control input is yielded using the error signal $\operatorname{err}(\boldsymbol{k})$ between target output pressure $\boldsymbol{P}_{\boldsymbol{r}}(\boldsymbol{k})$ and the present output pressure $\boldsymbol{P}_{\boldsymbol{o}}(\boldsymbol{k})$ of the optical servo valve. The input $\boldsymbol{u}(\boldsymbol{k})$ is as follows.

$$
\begin{gathered}
u(k)=K_{p} \cdot \operatorname{err}(k)+K_{d} \cdot(\operatorname{err}(k)-\operatorname{err}(k-1)) \\
+K i \cdot \sum_{i=0}^{k} \operatorname{err}(i) \\
\operatorname{err}(k)=P_{r}(k)-P_{o}(k) \\
K_{p}: \text { Proportional gain } \\
K_{d}: \text { Derivative gain } \\
K_{i}: \text { Integral gain }
\end{gathered}
$$

The position range of spool in the alignment operation of servo valve output pressure is very narrow section near a center position. Therefore, when the large proportionality gain was set up in the initial state, the spool moves up to end, then output pressure becomes oscillatory and does not settle to the target value.

Then, the PID control range is adjusted. The PID control is performed within the limits, and Bang-Bang control is performed out of this range. It is shown in Figure9. 


\subsection{Experiment result}

The control experiment to the rectangular wave of the target pressure $100[\mathrm{kPa}]$ is conducted. The experiment result is shown in Figure10.

As the result, the lag time is 0.20 [s] and setting time is 1.16 [s]. It is shown that the optical control of the pressure can be realized.

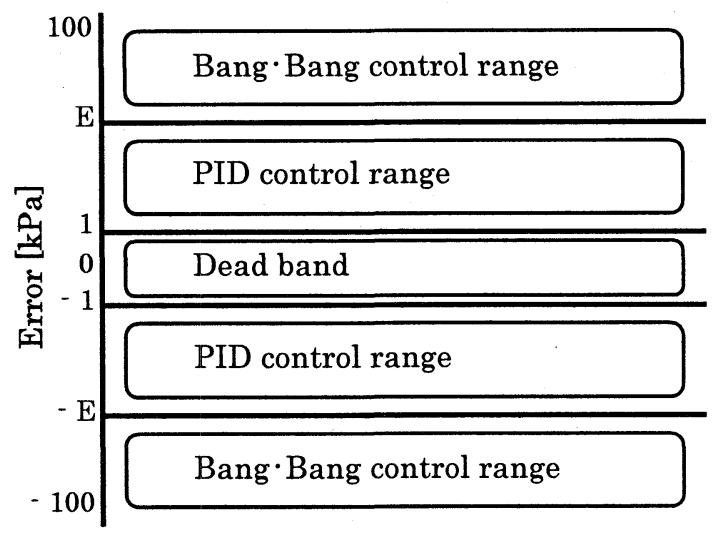

Figure9 Illustration of PID control band

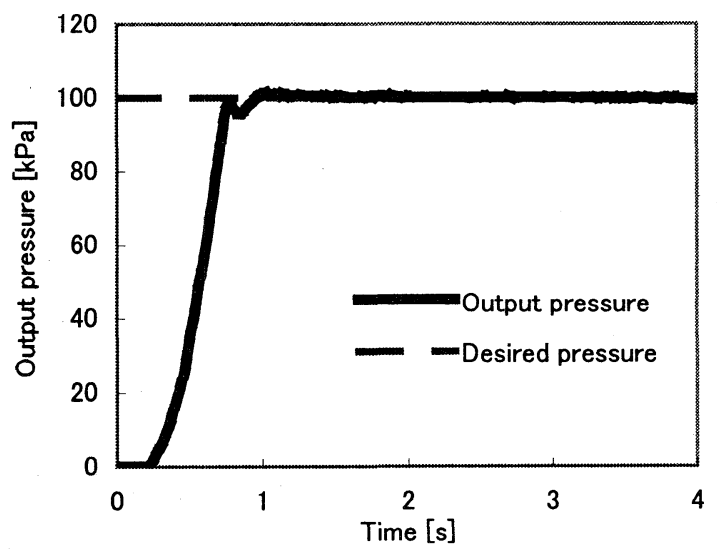

Figure10 Step response of optical servo valve

by PID \& Bang $\cdot$ Bang control

\section{Conclusions}

This research developed the "optical servo valve" which combined the opt-fluidic converter with the air pilot servo valve, and the investigations of the characteristic of this component are shown. Also the controlled output pressure by PID control is reported.

As a result obtained by comparison with this 3-stage of LPA cascade and 4-stage of LPA cascade, it is better to adopt 4-stage cascade in controlling the valve than that of conventional study.

Moreover, if each unit of an optical servo valve system is unified one, and if it is possible to develop an optical servo valve unit, the performance will be able to improve.

From now on, it is thought that clarifying the characteristic of an optical servo valve more and improving system configuration and control method attain the application to various pneumatic systems.

\section{Acknowledgment}

We were supported with Kuroda Precision Industries in the air servo valve system distribution.

Thanks for Kuroda Precision Industries.

\section{References}

1.Gurney,J.O., Photofluidic Interface, Trans. ASME, J.Dyn.Meas. \& Cont., 1984 106, 90.

2. Yamamoto,K.,

Characteristics

of a Opt-Fluidic Converter Utilizing Photo acoustic Effect, Trans. SICE Japan, 1991, 27-12, pp1405-1411.

3.Hockaday,B., Glomb,W., Taylor,K. and Waters,J., A Novel Opto-Fluidic Interface, J.Fluid Control, 1986 16-2/3,17.

4.Dohta,S. and Takamori,T.,Control of Attached Jet by Light, Trans. SICE, Japan, 1990. 